\title{
A influência da educação permanente na manipulação de cateteres vesicais de demora
}

The influence of permanent education in the manipulation of delay vesical catheters

La influencia de la educación permanente en la manipulación de catéteres vesicales

retardados

Tamires de Nazaré Soares ${ }^{1}$, Cleise Ellen Ferreira Pantoja ${ }^{2 *}$, Leandro Lopes Gibson Alves ${ }^{1}$, Lorrana Lopes Gibson Alves ${ }^{1}$, Alfredo Hiago Mota Gomes ${ }^{1}$, Thais Hetierre Abreu Monteiro ${ }^{1}$, Milene de Andrade GouvêaTyll ${ }^{3}$.

\section{RESUMO}

Objetivo: Analisar a influência da Educação Permanente na utilização das técnicas assépticas na passagem e manipulação de Cateteres Vesicais de Demora pela equipe de enfermagem em Unidades de Terapia Intensiva (UTIs). Métodos: Trata-se de uma pesquisa quantitativa realizado em duas Unidades de Terapia Intensiva adulto, de um Hospital Particular no Estado do Pará. Os dados foram coletados através de questionários para 15 enfermeiros e para os 35 técnicos de enfermagem Resultados: Sobre as respostas ao questionário antes do processo da Educação Permanente, na comparação entre o respondido pelos enfermeiros e técnicos, houve diferença significativa nas respostas em duas perguntas: Sobre lavagem das mãos em procedimentos invasivos e diante de furo na bolsa coletora de urina. Após a Educação Permanente, só houve desvio da resposta correta no uso do "tipo de luva na passagem do Cateter Vesical de Demora", e referentes as demais perguntas houve 100\% de acertos para ambas as categorias de profissionais. Conclusão: Os resultados deste estudo revelam o quanto a Educação Permanente é de extrema importância para a qualidade do serviço em saúde, pois proporciona que os erros recorrentes sejam evitados ou eliminados no ambiente assistencial.

Palavras-chave: Enfermagem, Educação permanente, Unidade de terapia intensiva.

\begin{abstract}
Objective: To analyze the influence of Permanent Education on the use of aseptic techniques in the passage and manipulation of Bladder Catheters by the nursing team in Intensive Care Units (ICUs). Methods: This is a quantitative research carried out in two adult Intensive Care Units, in a Private Hospital in the State of Pará. Data were collected through questionnaires for 15 nurses and 35 nursing technicians. Results: About the responses to questionnaire before the Permanent Education process, in the comparison between the answers answered by nurses and technicians, there was a significant difference in the answers in two questions: About hand washing in invasive procedures and before a hole in the urine collection bag. After Permanent Education, there was only a deviation from the correct answer in the use of the "type of glove in the passage of the Bladder Catheter", and regarding the other questions, there were $100 \%$ correct answers for both categories of professionals. Conclusion: The results of this study reveal how much Permanent Education is extremely important for the quality of the health service, as it provides that recurrent errors are avoided or eliminated in the care environment.
\end{abstract}

Keywords: Nursing, Continuing education, Intensive care unit.

1 Universidade da Amazônia, Belém - PA.

2 Universidade Federal do Pará, Belém - PA. *E-mail: ecleise@yahoo.com.br

${ }^{3}$ Fundação Pública Hospital de Clínicas Gaspar Viana, Belém - PA.

SUBMETIDO EM: 7/2020 ｜ＡCEITO EM: 7/2020｜ＰUBLICADO EM: 10/2020 


\section{RESUMEN}

Objetivo: analizar la influencia de la educación permanente en el uso de técnicas asépticas en el paso y manipulación de las sondas vesicales por parte del equipo de enfermería en las unidades de cuidados intensivos (UCI). Métodos: Esta es una investigación cuantitativa realizada en dos Unidades de Cuidados Intensivos para adultos, en un Hospital Privado en el Estado de Pará. Los datos fueron recolectados a través de cuestionarios para 15 enfermeras y 35 técnicos de enfermería. Resultados: Acerca de las respuestas a Cuestionario previo al proceso de Educación Permanente, en la comparación entre las respuestas respondidas por enfermeras y técnicos, hubo una diferencia significativa en las respuestas en dos preguntas: sobre el lavado de manos en procedimientos invasivos y ante un agujero en la bolsa de recolección de orina. Después de la educación permanente, solo hubo una desviación de la respuesta correcta en el uso del "tipo de guante en el paso del catéter vesical", y con respecto a las otras preguntas, hubo respuestas 100\% correctas para ambas categorías de profesionales. Conclusión: Los resultados de este estudio revelan cuánta educación permanente es extremadamente importante para la calidad del servicio de salud, ya que establece que los errores recurrentes se evitan o eliminan en el entorno de atención.

Palabras clave: Enfermería, Educación continua, Unidad de cuidados intensivos.

\section{INTRODUÇÃO}

A assistência aplicada em Unidade de Terapia Intensiva (UTI) requer procedimentos especializados para favorecer o prolongamento da sobrevida do paciente em situações adversas. Este fenômeno é altamente positivo por um lado, por outro é um dos fatores determinantes do aumento do risco de Infecção Hospitalar em pacientes críticos (HESPANHOL LAB, et al., 2019).

Infecção relacionada aos serviços de saúde é aquela adquirida após a entrada do paciente no hospital, ou seja, é toda e qualquer infecção manifestada no paciente após a sua entrada em um estabelecimento hospitalar e/ou durante a internação ou até mesmo depois de sua alta, podendo também ser relacionada diretamente a procedimentos intra-hospitalar (MENEGUETI MG, et al., 2012). As Infecções que afetam o trato urinário (ITU) consistem na proliferação de microrganismos (bactérias), no trato urinário, capazes de provocar lesões com gravidade variáveis. Estas infecções podem se instalar em quatro entidades clínicas distintas, de acordo com a localização anatômica da incidência, mantendo, todavia, relações entre elas: bacteriúria assintomática, uretrite, cistite e pielonefrite (JORGE HMS e VIDAL ECF, 2014).

As ITUs complicadas, são aquelas que estão associadas a condições que facilitem o risco de infecção, correspondendo às infecções sintomáticas que possuem como uma das principais causas a presença de corpos estranhos (REZENDE J, et al., 2016). Segundo Paschoal MRD e Bomfim FRC (2012), as ITUs são provocadas principalmente pela utilização do cateter na uretra, tendo em vista as modificações da resposta inflamatória, com supressão dos mecanismos de defesa inatos da mucosa uretral somado à aderência microbiana ao material do cateter.

A cateterização vesical é um procedimento amplamente utilizado, sendo de grande valor ao tratamento de variados processos patológicos. Seu uso é destinado a pacientes que apresentam incontinência urinaria, retenção urinaria, quando se faz necessária a avaliação exata de debito urinário, restrições pós-operatórias, coleta de amostra de urina, irrigação de bexiga ou instalação de medicamentos e nas cirurgias urológicas (MAZZO A, et al., 2015).

Os clientes sujeitados aos procedimentos invasivos como a cateterização vesical de demora estão mais suscetíveis a contrair a ITU, e na ocorrência de obstrução do trato urinário ocorre à reserva urinária criandose condições propicias à proliferação de microrganismos (PASCHOAL MRD e BOMFIM FRC, 2012). O processo de cateterização urinário é considerado um procedimento invasivo que consiste na introdução de um cateter via uretral até a bexiga do paciente, com a finalidade a drenagem de urina em pacientes com algum problema de eliminação da mesma. A cateterização pode ser caracterizada por sistema aberto (intermitente ou alívio), fechado (demora) e por via suprapúbica (ALMEIDA MM, et al., 2016). 
As infecções referentes a cateterização vesical, indicam um grande problema de saúde pública no Brasil, tanto para os pacientes, ressaltado pelo elevado números de infecções, quanto para a sociedade pelas graves consequências, como os altos custos hospitalares para tratar tais infecções (MAGALHÃES SR, et al., 2014). Entre os setores hospitalares que mais se destacam nos índices de infecções é a UTI, tornandose cada vez mais frequentes devido ao longo período de internação de cada paciente, expondo-o por mais tempo aos agentes infecciosos bem como a diminuição da resposta imune, e devido aos procedimentos invasivos realizados característicos do setor, o que tornam os pacientes ainda mais susceptíveis aos processos infecciosos (GUIMARÃES JFS, 2014).

Para Silva JP, et al. (2014), os estudos relatam que o cateterismo vesical, deve ser realizado por um profissional treinado e capacitado através de embasamento teórico e científico para realizar técnica asséptica e correta inserção. Diante do exposto, torna-se necessário a participação no treinamento de toda a equipe envolvida nos cuidados e manutenção do cateter urinário com relação à prevenção de ITU, incluindo alternativas de manejo e remoção, já que este é um profissional que participa ativamente na realização do cateterismo vesical. Apesar dos avanços e medidas estabelecidas em protocolos e portarias para a prevenção e controle das infecções urinarias, a baixa adesão pelos profissionais às recomendações, vem sendo um desafio para as instituições de saúde, devido à falta de conhecimento ou à dificuldade de mudança nos hábitos sedimentados ao longo da vida (PASCHOAL MRD e BOMFIM FRC, 2012).

Com base em publicações científicas e considerando o papel fundamental da enfermagem na recuperação clínica, prevenção de agravos e recuperação dos clientes em uma UTI, e que há a necessidade que os profissionais tenham a consciência da importância de uma educação permanente para as medidas preventivas vinculada a ITU, pois estas proporcionarão ao cliente segurança e qualidade no serviço, no qual há problemas na efetivação das ações de EPS no que consta à prática de capacitação dos profissionais de enfermagem que buscam atender as necessidades de mudanças nas práticas de saúde conforme os princípios do Sistema Único de Saúde (PINTO JR, et al., 2015).

Para se adaptarem a realidade vivenciada, as instituições de saúde e serviços de enfermagem buscam a educação permanente como parte fundamental para aperfeiçoamento dos profissionais, e diante disso, o objetivo geral da pesquisa é analisar a influência da Educação Permanente (EP) na utilização das técnicas assépticas na passagem e manipulação de Cateteres Vesicais de Demora (CVD) pela equipe de enfermagem em Unidades de Terapia Intensiva (UTIs) adulta de um hospital particular de Belém, Pará.

\section{MÉTODOS}

Tratar-se de uma pesquisa quantitativa, descritiva e transversal aprovada pelo Comitê de Ética da Universidade da Amazônia (UNAMA), com o parecer 2.074.738. O presente estudo foi realizado em duas Unidades de Terapia Intensiva Adulto, de um Hospital Particular que é referência do plano de saúde no munícipio de Belém no Estado do Pará. A escolha da área especifica se deu devido a complexidade do local diante da assistência com o paciente necessitando de cuidados no manuseio com os equipamentos e procedimentos realizados.

Para a coleta dos dados, utilizaram dois questionários com perguntas abertas e fechadas, direcionado para os enfermeiros e técnicos de enfermagem, e a partir das respostas, foram caracterizados os níveis de conhecimento sobre o tema abordado de cada profissional. Os participantes que acertaram um quantitativo menor ou igual à seis $(\leq 6)$ questões foram enquadrados com nível regular, de sete e oito $(7$ e 8$)$ nível bom e de nove e dez (9 e 10) excelente.

Foi realizado uma palestra no auditório do próprio hospital da pesquisa com o tema: "A importância da educação permanente para a cateterização eficiente e asséptica em Unidades de Terapia Intensiva (UTIs)", tendo a duração de 60 minutos, ministrada nos três turnos. Fizeram parte da pesquisa 50 trabalhadores, sendo destes quinze 15 enfermeiros e trinta e cinco 35 técnicos de enfermagem que atuam nas UTIs, sendo disponibilizados para os profissionais participantes que integraram a pesquisa, o Termo de Consentimento Livre e Esclarecido (TCLE), para assegurar a privacidade e sigilo dos dados dos participantes e os mesmos foram identificados por números. O período do estudo foi de janeiro a junho de 2017. 
Para a determinação da estabilidade e confiabilidade utilizamos o teste de Kappa. Trata-se de um teste não-paramétrico destinado a distinguir as proporções da mesma variável mensurada a nível nominal em dois momentos distintos, antes e depois, testando a reprodutibilidade dos resultados, admitindo que haja harmonia nas dimensões das respostas nos dois períodos considerados. As informações são inseridas sob a forma de uma tabela de contingência $2 \times 2$.

Os dados coletados foram apurados em uma matriz no formato de planilha eletrônica no software Microsoft Excel. Depois da validação dos dados, ocorreu a transferência dos mesmos para o software Statistical Package for Social Sciences (SPSS) for Windows, versão 20.0 e foram submetidos à análise descritiva para obtenção das frequências absolutas e relativas das variáveis analisadas.

Para avaliação da significância entre os gêneros e tempo de atuação segundo categoria profissional, bem como para a comparação entre as respostas dos grupos de observação entre os momentos de coleta (antes e depois do processo de educação), bem como para averiguação do nível de conhecimento referente ao uso de técnicas assépticas na CVD antes e depois da atividade de educação permanente segundo categoria profissional; foi utilizado para a análise o Teste Exato de Fisher ou pelo Teste G, se a tabulação tinha, respectivamente, dupla entrada (duas colunas e duas linhas) ou maior tabulação no software BioEstat versão 5.0 .

$\mathrm{Na}$ análise bivariada, verificou-se a independência entre cada uma das variáveis (respostas) entre os momentos de coleta (antes e depois do processo de educação permanente) nos grupos de observação. Para esta análise, as respostas foram categorizadas em "correta" e "incorreta". Nesta situação, foi realizada análise pelo teste McNemar no software BioEstat versão 5.0. Adicionalmente foi realizada a análise de concordância das respostas entre os dois momentos pelo teste Kappa, no software SPSS.

\section{RESULTADOS}

\section{Frequência do Gênero e Tempo de Atuação Segundo Categoria Profissional}

A comparação nesta tabela é entre enfermeiro e técnicos de enfermagem. Quanto ao gênero, é evidente que há diferença na distribuição, pois não houve técnicos do gênero masculino, apresentando diferença estatisticamente. Em relação ao tempo de atuação, os técnicos apresentaram maiores proporções nas faixas de tempo mais elevadas (Tabela 1).

Tabela 1 - Frequência do gênero e tempo de atuação segundo categoria profissional.

\begin{tabular}{|c|c|c|c|c|c|c|c|}
\hline \multirow{2}{*}{ Variáveis } & \multicolumn{2}{|c|}{ Enfermeiro } & \multicolumn{2}{|c|}{ Técnico } & \multicolumn{2}{|c|}{ Total } & \multirow{2}{*}{$p^{* * *}$} \\
\hline & $\mathrm{n}^{*}$ & $\%$ ** & $\mathrm{n}^{\star}$ & $\%$ \%* & $\mathrm{n}^{*}$ & $\%$ \%* & \\
\hline \multicolumn{8}{|l|}{ Gênero } \\
\hline Feminino & 11 & 73,3 & 35 & 100,0 & 46 & 92,0 & \multirow{2}{*}{0,006} \\
\hline Masculino & 4 & 26,7 & 0 & 0,0 & 4 & 8,0 & \\
\hline \multicolumn{8}{|c|}{ Tempo de atuação profissional } \\
\hline 1 a 5 anos & 7 & 46,7 & 8 & 22,9 & 15 & 30,0 & \multirow{4}{*}{0,004} \\
\hline 6 a 10 anos & 8 & 53,3 & 11 & 31,4 & 19 & 38,0 & \\
\hline 11 a 15 anos & 0 & 0,0 & 9 & 25,7 & 9 & 18,0 & \\
\hline 16 a 20 anos & 0 & 0,0 & 7 & 20,0 & 7 & 14,0 & \\
\hline Total & 15 & 100,0 & 35 & 100,0 & 50 & 100,0 & ----- \\
\hline
\end{tabular}

Fonte: Soares TN, et al., 2020.

\section{Frequências das Respostas das Questões Referentes ao Uso De Técnicas Assépticas Na CVD Antes da Educação Permanente por Categoria Profissional}

Sobre as respostas ao questionário antes do processo da educação permanente, na comparação entre o respondido pelos enfermeiros e técnicos, houve diferença significativa $(p<0,05)$ nas respostas em duas perguntas: a) Sobre lavagem das mãos em procedimentos invasivos, os técnicos $31(85,5 \%)$ relataram "lavar antes e depois" em maior frequência que os enfermeiros $6(40,0 \%)$, e; b) Diante de furo na bolsa coletora de urina, os técnicos $30(85,7 \%)$ concordaram que o certo seria a "troca de todo o sistema" em maior proporção que os enfermeiros, pois somente $8(53,3 \%)$ relataram a "troca de todo o sistema" (Tabela 02). 
Tabela 2 - Frequências das respostas das questões referentes ao uso de técnicas assépticas na CVD antes da educação permanente, por categoria profissional.

\begin{tabular}{|c|c|c|c|c|c|c|c|c|}
\hline \multirow{2}{*}{\multicolumn{2}{|c|}{ Variáveis }} & \multicolumn{2}{|c|}{ Enfermeiro } & \multicolumn{2}{|c|}{ Técnico } & \multicolumn{2}{|c|}{ Total } & \multirow{2}{*}{$p^{\star \star *}$} \\
\hline & & $\mathbf{n}^{*}$ & $\%$ \%* & $\mathbf{n}^{\star}$ & $\%$ \%* & $\mathbf{n}^{\star}$ & $\%$ \%* & \\
\hline \multirow{44}{*}{ 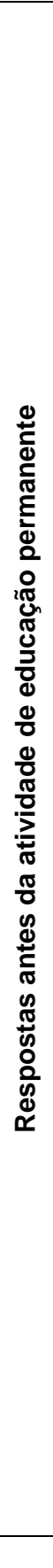 } & \multicolumn{7}{|c|}{2 Importância da lavagem das mãos em procedimento invasive } & 0,001 \\
\hline & Antes & 2 & 13,3 & 3 & 8,6 & 5 & 10,0 & \\
\hline & Depois & 7 & 46,7 & 0 & 0,0 & 7 & 14,0 & \\
\hline & Antes e depois & 6 & 40,0 & 31 & 88,6 & 37 & 74,0 & \\
\hline & NDA & 0 & 0,0 & 1 & 2,9 & 1 & 2,0 & \\
\hline & \multicolumn{7}{|c|}{3 Luva utilizada na passagem e manutenção do Sistema de CVD } & 0,528 \\
\hline & Luva de procedimento & 6 & 40,0 & 13 & 37,1 & 19 & 38,0 & \\
\hline & Luva estéril & 9 & 60,0 & 19 & 54,3 & 28 & 56,0 & \\
\hline & Não usa & 0 & 0,0 & 0 & 0,0 & 0 & 0,0 & \\
\hline & Não faço o procedimento & 0 & 0,0 & 3 & 8,6 & 3 & 6,0 & \\
\hline & \multicolumn{7}{|c|}{4 Atitude diante de furo na bolsa coletora de urina } & 0,001 \\
\hline & Tampa furo com esparadrapo & 1 & 6,7 & 0 & 0,0 & 1 & 2,0 & \\
\hline & Troca todo o Sistema & 8 & 53,3 & 30 & 85,7 & 38 & 76,0 & \\
\hline & Troca somente sonda & 6 & 40,0 & 1 & 2,9 & 7 & 14,0 & \\
\hline & Avisa família para providenciar a troca & 0 & 0,0 & 4 & 11,4 & 4 & 8,0 & \\
\hline & \multicolumn{7}{|c|}{5 Tempo de permanência de CVD no paciente em UTI } & 0,990 \\
\hline & 15 a 30 dias & 13 & 86,7 & 29 & 82,9 & 42 & 84,0 & \\
\hline & 30 a 45 dias & 2 & 13,3 & 6 & 17,1 & 8 & 16,0 & \\
\hline & 45 a 60 dias & 0 & 0,0 & 0 & 0,0 & 0 & 0,0 & \\
\hline & 60 a 90 dias & 0 & 0,0 & 0 & 0,0 & 0 & 0,0 & \\
\hline & \multicolumn{7}{|c|}{6 Procedimento diante limiar de uso de bolsa coletora } & 0,141 \\
\hline & Esvaziamento imediato & 15 & 100,0 & 28 & 80,0 & 43 & 86,0 & \\
\hline & Troca só no próximo plantão & 0 & 0,0 & 4 & 11,4 & 4 & 8,0 & \\
\hline & Avisa família para que esvazie & 0 & 0,0 & 2 & 5,7 & 2 & 4,0 & \\
\hline & Solicito a outro enfermeiro & 0 & 0,0 & 1 & 2,9 & 1 & 2,0 & \\
\hline & \multicolumn{7}{|c|}{7 Produto usado na assepsia diante da passagem de cateter } & 0,539 \\
\hline & Clorexidina degermante & 12 & 80,0 & 33 & 94,3 & 45 & 90,0 & \\
\hline & Água destilada & 3 & 20,0 & 2 & 5,7 & 5 & 10,0 & \\
\hline & Soro fisiológico & 0 & 0,0 & 0 & 0,0 & 0 & 0,0 & \\
\hline & Lidocaína em gel & 0 & 0,0 & 0 & 0,0 & 0 & 0,0 & \\
\hline & \multicolumn{7}{|l|}{8 Material usado na passagem de CVD } & 0,143 \\
\hline & Conjunto 1 & 0 & 0,0 & 1 & 2,9 & 1 & 2,0 & \\
\hline & Conjunto 2 & 10 & 66,7 & 14 & 40,0 & 24 & 48,0 & \\
\hline & Conjunto 3 & 0 & 0,0 & 4 & 11,4 & 4 & 8,0 & \\
\hline & Conjunto 4 & 5 & 33,3 & 16 & 45,7 & 21 & 42,0 & \\
\hline & \multicolumn{7}{|c|}{9 Consideração da importância da Educação Permanente } & 1,000 \\
\hline & Sim & 15 & 100,0 & 35 & 100,0 & 50 & 100,0 & \\
\hline & Não & 0 & 0,0 & 0 & 0,0 & 0 & 0,0 & \\
\hline & \multicolumn{7}{|c|}{10 Tempo ideal para realização de ações de educação permanente } & 0,237 \\
\hline & 15 a 30 dias & 7 & 46,7 & 25 & 71,4 & 32 & 64,0 & \\
\hline & 30 a 45 dias & 7 & 46,7 & 7 & 20,0 & 14 & 28,0 & \\
\hline & 45 a 60 dias & 1 & 6,7 & 2 & 5,7 & 3 & 6,0 & \\
\hline & 60 a 90 dias & 0 & 0,0 & 1 & 2,9 & 1 & 2,0 & \\
\hline & Total & 15 & 100,0 & 35 & 100,0 & 50 & 100,0 & |----- \\
\hline
\end{tabular}

Fonte: Soares TN, et al., 2020

\section{Frequências das Respostas das Questões Referentes ao Uso de Técnicas Assépticas na CVD depois da Educação Permanente por Categoria Profissional}

Após o processo de Educação Permanente, só houve desvio da resposta correta no uso do tipo de luva na passagem do CVD, pois a correta seria a estéril, mesmo assim em baixa frequência do total de participantes. Desta forma $48(96,0 \%)$ participantes acertaram a pergunta. Referentes as demais perguntas houve $100 \%$ de acertos para ambas as categorias de profissionais (Tabela $\mathbf{3}$ ). 
Tabela 3 - Frequências das respostas das questões referentes ao uso de técnicas assépticas na CVD depois da atividade de educação permanente, por categoria profissional.

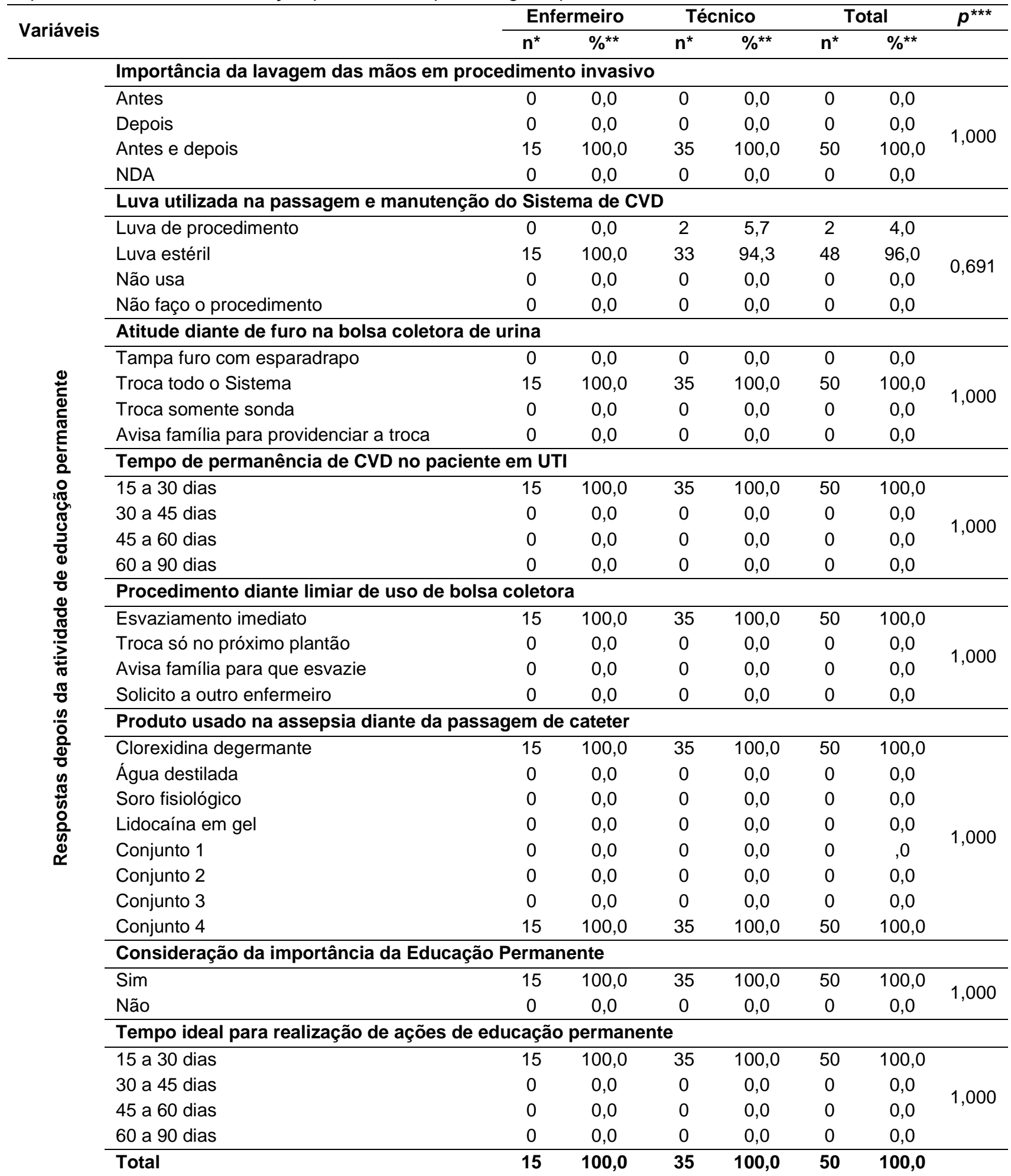

Fonte: Soares TN, et al., 2020.

\section{DISCUSSÃO}

Os resultados da pesquisa apontam um índice maior do gênero feminino em relação aos profissionais de enfermagem do estudo, com 46 participantes $(92,0 \%)$ e apenas 4 participantes $(8,0 \%)$ sendo do gênero masculino, apresentando então a relevância de mulheres principalmente na categoria de técnicos de 
enfermagem. Logo adiante, no corpo do estudo, evidencia-se na tabela 02 , a necessidade de mudanças no conjunto de práticas do cuidado principalmente quanto às medidas assépticas e mendicidade de aperfeiçoamento do conhecimento científico associado á prática quanto às técnicas conduzidas pelos enfermeiros e técnicos para a prevenção de infecção ao CVD.

Dentre os procedimentos invasivos na UTI, o mais comum é a cateterização vesical, e quando não é manuseado de forma adequada antes mesmo de sua inserção, transforma-se em um cateter contaminado com microorganismo, facilitando ao início de um processo infeccioso desde o momento da introdução do mesmo. As infecções causadas por este procedimento relacionadas às falhas nas técnicas assépticas durante o procedimento se tornam uma complicação grave, no qual os microorganismos que colonizam a região periuretral penetram na bexiga do paciente que se encontra vulnerável (MIRANDA AL, 2016).

Os dados obtidos da pesquisa mostram que a higienização das mãos se apresenta como uma falta de adesão dos profissionais quanto à adequação das técnicas antes da Educação permanente. Diante desse contexto, Ferreira LL, et al. (2019), apresenta em sua revisão de literatura que a técnica correta da higienização das mãos em um setor crítico se torna fundamental antes e após os procedimentos, no qual é um ambiente que facilita a exposição a agentes infecciosos, podendo se tornar um grave problema ao risco de piora clínica e consequentemente um maior tempo de permanência no ambiente hospitalar. Além disso, ressalta a problemática na não adesão do profissional que necessita de mudança de comportamento na assistência à saúde.

Resende J, et al. (2016), em sua pesquisa demonstra que entre os agentes etiológicos que mais se destacam como causa de Infecção do Trato Urinário e que estão relacionadas a inadequação da higienização das mãos são a Pseudomonas aeruginosa que afetam principalmente pacientes internados em unidades especializadas, sendo responsável por infecções oportunistas em pacientes com defesas imunológicas comprometidas.

Observa-se também em um estudo de Merces MC, et al. (2013), a relevância do procedimento de passagem de Cateteres Vesicais quanto aos materiais, em saber manuseá-los de forma eficiente, que correlaciona com os dados da pesquisa quanto ao uso de luva estéril e troca de todo o sistema do CVD, por exemplo; pois na maioria das vezes os pacientes críticos devido a complexidade de sua patologia, além das intercorrencias que eles estão sujeitos a apresentar nesse ambiente, acaba contribuindo fortemente para a ocorrência da ITU, favorecendo o aumento da morbidade e da mortalidade dos pacientes internados, o que acaba sendo um evento adverso evitável.

Achados esses que corroboram os estudos de Ferreira LL, et al. (2019), que também identifica os principais cuidados com pacientes com CVD, enfatizando que as medidas assépticas devem ser praticadas desde a inserção até a retirada do cateter, que reflete nos resultados da pesquisa quanto a utilização de luva estéril, cuidado com a bolsa coletora, tempo de permanência e materiais usados no procedimento, conforme vimos na Tabela 2 da pesquisa, pois no que diz respeito a esse procedimento, pode haver 0 desenvolvimento de um biofilme que serve como capa protetora das bactérias, dificultando sua eliminação.

No ambiente em que se encontra o cliente grave, o uso de tecnologia invasiva muitas vezes é o principal recurso para estabilizar o paciente hemodinamicamente instável. Entretanto, para que o cliente consiga se beneficiar dessas tecnologias invasivas, é de relevância que os profissionais de saúde estejam muito bem capacitados e ciente da extensão e gravidade da patologia do doente submetido à terapia intensiva para evitar surgimento de infecções (PAIVA PA, et al., 2015).

No decorrer da pesquisa, observa-se também um item relevante, que aparece como falha nas respostas dos participantes, no que se refere ao uso de luvas estéreis. Salienta-se que as luvas funcionam como barreira e disseminação de microrganismos nos procedimentos invasivos, além de ser uma medida profilática para o profissional de saúde (SOUZA ELV, et al., 2011).

A correta técnica de calçamento é fundamental para não contaminar a luva estéril, e a sua retirada é fundamental no sentido de não contaminar o profissional com o conteúdo externo das luvas, que é risco potencial de sangue e fluidos corporais contaminados (SOUZA LM, et al., 2015). Além disso, é importante ressaltar que infecção hospitalar é aquela adquirida dentro do serviço de saúde, principalmente nas UTIs, e 
pode ser transmitida do profissional para o paciente, se não adotadas as devidas medidas de proteção e técnicas corretas. A problemática continuará a partir do momento que os profissionais responsáveis pela passagem e manutenção desses cateteres não utilizarem as técnicas assépticas recomendadas e não procurarem atualização e capacitações de protocolos através da educação permanente (EP) do referido hospital (TORRES RA e TORRES BR, 2015).

As Infecções do trato urinário estão cada vez mais presentes nos setores críticos. As recomendações para o seguimento de protocolos na realização de procedimentos quanto a ambiente hospitalar se tornam marco na qualidade nos serviços de saúde, que é fundamental no cuidado de enfermagem (TORRES RA e TORRES BR, 2015).

Massaroli A (2014), enfatiza que essas técnicas básicas corretas devem ser incentivadas permanentemente. A qualificação e reciclagem podem ser ofertadas através do programa de Educação Permanente para os profissionais da enfermagem que de forma direta ou indireta manipulam os cateteres vesicais de demora e é indicada como uma maneira efetiva e essencial de prevenção das infecções relacionadas ao serviço em saúde, o que está evidenciado em nossas pesquisas, pois a Educação Permanente se mostrou uma ferramenta estratégica para qualidade da assistência

Foi observado nos estudos de Cardoso SAC e MAIA LFS (2014), a finalidade das técnicas padronizadas é diminuir ao máximo a transmissão de microrganismos a partir de fontes de infecção conhecidas ou desconhecidas. As precauções Padrão enfatizam condutas como, higienização das mãos, uso de luvas, máscaras, protetor facial e de olhos, roupas de proteção, manuseio cuidadoso dos instrumentos perfurocortantes e cuidado com matérias e equipamentos. No entanto, com a prática da Educação Permanente, a realidade foi quase totalmente invertida, pois $100,0 \%$ dos participantes acertaram a pergunta sobre a lavagem das mãos, mas $4 \%$ dos técnicos erraram sobre a luva a ser utilizada para o CVD enquanto que $96,0 \%$ acertaram.

Diante disso, os achados da pesquisa enfatizam a Educação Permanente como prática de qualificação, atualização e mudança pela compreensão dos erros decorrentes das práticas cotidianas, considerando que a conscientização por meio da integração teórica e prática agraciam à equipe de enfermagem com maior comprometimento e responsabilidade em executar os cuidados respaldados e fundamentados em um conhecimento científico (RUPOLO DJ, et al., 2014).

A finalidade das técnicas padronizadas é diminuir ao máximo a transmissão de microrganismos a partir de fontes de infecção conhecidas ou desconhecidas. As precauções Padrão enfatizam condutas como, higienização das mãos, uso de luvas, máscaras, protetor facial e de olhos, roupas de proteção, manuseio cuidadoso dos instrumentos perfuro-cortantes e cuidado com matérias e equipamentos (PAIVA PA, et al., 2015).

O manejo da ITU depende de medidas simples adotadas, como a conscientização dos profissionais da enfermagem, a realização e real indicação de procedimentos invasivos como a caterização vesical, o uso criterioso de antimicrobianos, a vigilância constante para detecção de novos casos, e a mais simples e importante, a técnica de lavagem das mãos, que está a cima de qualquer ação de prevenção por mais tecnológica que seja (QUEIRÓS MI, et al., 2011).

Os resultados da pesquisa mostram que a Educação Permanente é um artificio essencial que tem como objetivo melhorar o desempenho profissional, e se aplicada como um processo contínuo, possibilita à aquisição de conhecimentos, de técnicas e de atitudes corretas, para interagir e interferir na realidade além de minimizar os problemas e dentro desse âmbito, tem uma função essencial na educação desses trabalhadores, contribuindo e capacitando o mesmo a ser responsável pelo seu desenvolvimento e mudança (BEZERRA ALQ, et al., 2012).

A atualização do conhecimento deve ser constante entre os profissionais da saúde, fazendo-se necessário a criação e utilização de novas estratégias pelo enfermeiro frente ao novo modelo tecnológico vivido na atualidade, fazendo-se necessário uma reciclagem constante frente a esse modelo. Existe na atualidade, uma mudança constante e acelerada em relação aos conhecimentos e saberes tecnológicos na 
área da saúde, que de certa forma acaba desprivilegiando ações da prática diárias do enfermeiro e das áreas especificas da saúde (ALMEIDA JRS, et al., 2016).

Os profissionais enfermeiros de uma UTI, necessitam estar capacitados para o exercício de atividade de alta complexidade, fazendo-se necessário uma autoconfiança, além do conhecimento com embasamento científico para o atendimento do paciente com segurança, consideradas meios de extrema importância para o alcance dos resultados esperados. Os aparatos tecnológicos são considerados de extrema importância, porém a maior diferencial no mercado competitivo são as pessoas. Com isso, o processo de qualificação do enfermeiro é considerado de grande valia, na garantia da qualidade da assistência ao paciente (MERCES MC, et al., 2013).

\section{CONSIDERAÇÕES FINAIS}

Os resultados deste estudo revelam o quanto a Educação Permanente (EP) é de extrema importância para a qualidade do serviço em saúde, pois proporciona que os erros recorrentes sejam evitados ou eliminados no ambiente assistencial, que antes da EP diversos profissionais participantes deste estudo responderam incorretamente a algumas perguntas, e isso exige do profissional o desenvolvimento contínuo de aprendizagem, autonomia. Contudo reafirmamos a importância da EP ao ser percebido $100 \%$ de assertividade das respostas dos profissionais após as palestras efetuadas, diante das mesma perguntas realizadas antes da execução das atividades da EP. Acerca disso há uma real necessidade de promover a EP para toda a equipe enfermagem, permitindo uma qualificação constante, atualização e mudança pela visão dos erros ainda vigentes da prática do dia-a-dia, considerando que a compressão por meio da associação teórica e prática conferem à equipe de profissionais da enfermagem maior comprometimento e responsabilidade em praticar o cuidado respaldado e fundamentado em um conhecimento científico. A vigilância é essencial para verificar o seguimento de protocolos e práticas e os resultados da Educação Permanente oportunizam um momento de reflexão importante profissionais de saúde quanto às práticas de segurança do paciente.

\section{REFERÊNCIAS}

1. ALMEIDA MM, et al. Evidências na prática de cateterismo urinário. Rev. Facema. 2016; 2(2):196-201.

2. ALMEIDA JRS, et al. Educação Permanente em Saúde: uma estratégia para refletir sobre o processo de trabalho. Revista da ABENO. 2016, 16 (2): 7-15.

3. ALVES AA. Fatores predisponentes para ocorrência de infecção do trato urinário associada ao uso de cateter vesical de demora. Trabalho de Conclusão de Curso (Curso de Bacharelado em Enfermagem) - Centro de Ciências Biológicas e Saúde, Universidade Federal da Paraíba. 2014; 23 p.

4. BEZERRA ALQ, MUNARI DB. O processo de educação continuada na visão de enfermeiros de um hospital universitário. Rev. Eletr. Enf. 2012, 14(3):618- 25.

5. CARDOSO SAC, MAIA LFS. Cateterismo vesical de demora na UTI adulto: o papel do enfermeiro na prevenção de infecção do trato urinário. São Paulo: Revista Recien. 2014; 4(12):5-14.

6. FERREIRA LL, et al. Cuidado de enfermagem nas Infecções Relacionadas à Assistência à Saúde: Scoping review. Rev Bras Enferm. 2019, 72(2):498-505.

7. GUIMARÃES JFS. Atuação Do Enfermeiro no controle da infecção do trato urinário (ITU) na unidade de terapia intensiva (UTI). Atualiza Associação Cultural. Faculdade Metropolitana. 2014; 08 p.

8. HESPANHOL LAB, et al. Infecção relacionada à Assistência à Saúde em Unidade de Terapia Intensiva Adulto. Revista Eletrônica Enfermería Global. 2019, 20 (53): 229- 241.

9. JORGE HMS, VIDAL ECF. Infecção do trato urinário hospitalar e suas implicações para gestão do cuidar: revisão integrativa. Cad. Cult. Ciênc. 2014, 13 (1): 39-48.

10. MAGALHÃES SR, et al. Evidências para a prevenção de infecção no cateterismo vesical: revisão integrativa. Rev enferm UFPE on line. 2014, 8(4):1057-63.

11. MASSAROLI A, et al. Educação Permanente para o aperfeiçoamento do Controle de Infecção Hospitalar: revisão integrativa. Sau. \& Transf. Soc. 2014, 5(1): 07-15.

12. MAZZO A, et al. Cateterismo urinário de demora: prática clínica. Revista Eletrônica Enfermería Global. 2015,10 (38): $60-68$.

13. MENEGUETI MG, et al. Infecção urinária em Unidade de Terapia Intensiva: um indicador de processo para prevenção. Rev Rene. 2012; 13(3):632-8. 
14. MERCES MC, et al. A prática do (a) enfermeiro (a) na inserção do cateter de Folley em pacientes de unidade de terapia intensiva: limites e possibilidades. Rev Epidemiol Control Infect. 2013, 3(2):55-61.

15. MIRANDA AL, et al. Results after implementation of a protocol on the incidence of urinary tract infection in an intensive care unit. Rev. Latino-Am. Enfermagem. 2016, 24(2): 02- 09.

16. PASCHOAL MRD, BOMFIM FRC. Infecção do trato urinário por cateter vesical de demora. 2012, 16(6):213-226.

17. PAIVA PA, et al. Medidas de prevenção e controle das infecções hospitalares em unidade de terapia intensiva. Revista da Universidade Vale do Rio Verde, Três Corações. 2015, 13 (2): 669-680.

18. PINTO JR, et al. Educação permanente: reflexão na prática da enfermagem hospitalar. Tempus, actas de saúde colet, Brasília. 2015, 9(1): 155-165.

19. QUEIRÓS MI. Revista Rene. Infecções urinárias e uso de cateter vesical de demora em unidade pediátrica. Rev Rene, Fortaleza. 2011, 12(2):295-301.

20. RESENDE J, et al. Infecção do trato urinário de origem hospitalar e comunitária: visão dos principais microorganismo causadores e perfil de susceptibilidade. Revista Científica Fagoc Saúde. 2016; 1(1): 55-62.

21. RUPOLO DJ, et al. Infecções do trato urinário associadas ao uso de sonda vesical de demora: prevalência e susceptibilidade microbiana.Revista Eletrônica Gestão \& Saúde. 2014, 5(3): 992-05.

22. SILVA JP, et al. Intervenção de enfermagem na prevenção nas infecções do trato urinário relacionado ao cateterismo vesical de demora: uma revisão integrativa da literatura. Ciências biológicas e da saúde. 2014, 1(3):2133.

23. SOUZA LL. Ciencias \& Cognição. Representações de gênero na prática de enfermagem na perspectiva de estudantes. Ciências \& Cognição. 2014, 19(2): 218-232.

24. SOUZA ELV, et al. Uso dos equipamentos de proteção individual em unidade de terapia intensive. Revista de Enfermagem Referência. 2011, 3(4): 125-133.

25. SOUZA LMS, et al. Adesão dos profissionais de terapia intensiva aos cinco momentos da higienização das mãos. Rev Gaúcha Enferm. 2015 dez;36(4):21-8.

26. TORRES RA, TORRES BR. Importância e bases de um programa de controle e prevenção de infecção em unidade de terapia intensiva geral. Rev Med Minas Gerais. 2015; 25(4): 577-582. 